\title{
A Field-length based refinement criterion for adaptive mesh simulations of the interstellar medium (Research Note)
}

\author{
O. Gressel ${ }^{1,2}$ \\ 1 Astrophysikalisches Institut Potsdam, An der Sternwarte 16, 14482 Potsdam, Germany \\ e-mail: 0.gressel@qmul.ac.uk \\ 2 Astronomy Unit, School of Mathematical Sciences, Queen Mary, University of London, Mile End Road, London E1 4NS, UK
}

Received 1 September 2008 / Accepted 2 February 2009

\section{ABSTRACT}

\begin{abstract}
Context. Adequate modelling of the multiphase interstellar medium requires optically thin radiative cooling, containing an inherent thermal instability. The size of the resulting condensation and evaporation interfaces is determined by the so-called Field-length, which represents the dimension across which the instability is damped significantly by thermal conduction.

Aims. We study the relevance of conduction scale effects in the numerical modelling of a bistable medium and check the applicability of conventional and alternative adaptive mesh techniques.

Methods. The low value of the thermal conduction coefficient in the ISM defines a multiscale problem, promoting the use of adaptive meshes. We introduce a refinement strategy that applies the Field condition by Koyama \& Inutsuka as a refinement criterion. The described method is similar to the Jeans criterion for gravitational instability by Truelove and allows us to trace efficiently the unstable gas situated at the thermal interfaces.

Results. We present test computations that demonstrate the higher accuracy of our proposed refinement criterion compared to refinement based on the local density gradient. Apart from its usefulness as a refinement trigger, we do not find evidence in favour of the Field criterion being a prerequisite for numerical stability.
\end{abstract}

Key words. hydrodynamics - instabilities - ISM: general - ISM: structure

\section{Introduction}

The structure of the turbulent interstellar medium (ISM) of the Milky Way and other disk galaxies is determined by various complex physical processes. In particular, the formation of the neutral atomic phase of the ISM is believed to be regulated by condensation under the action of thermally bistable radiative cooling. The related thermal instability (TI) has been studied extensively as a modifying agent for various driving sources of turbulence in the ISM. Numerical studies have included the magneto-rotational instability (MRI) (Piontek \& Ostriker 2005, 2007), explosions of SNe (Korpi et al. 1999; de Avillez \& Breitschwerdt 2005; Joung \& Mac Low 2006; Gressel et al. 2008a,b), and converging flows (Audit \& Hennebelle 2005; Vázquez-Semadeni et al. 2006; Heitsch et al. 2008). Adaptive mesh MHD simulations of the last scenario were performed by Hennebelle et al. (2008), who applied a simple density threshold as a refinement criterion.

The development of TI alone was explored under various conditions by Sánchez-Salcedo et al. (2002). Moreover, there was a discussion of whether TI in conjunction with thermal conduction can act as an independent source of turbulence (Brandenburg et al. 2007; Koyama \& Inutsuka 2006).

In dynamic simulations of the ISM, the radiative cooling is usually treated in the limit of an optically thin plasma, i.e., radiative transport effects are neglected. The cooling rate is then prescribed by $\Lambda(T)$ in units of erg $\mathrm{cm}^{3} \mathrm{~s}^{-1} \mathrm{~g}^{-2}$. Here we adopt the cooling function from Sánchez-Salcedo et al. (2002) that is a piecewise power-law fit to results by Wolfire et al. (1995).
In this paper, we focus on an issue in the numerical treatment of thermally bistable gas first brought up by Koyama \& Inutsuka (2004). The comprehensive theoretical analysis by Field (1965) reveals that, in the case of vanishing thermal conduction, TI has a finite growth rate in the limit of high wave numbers. In the discrete representation of the fluid, this implies that numerical fluctuations on grid scale will be prone to artificial amplification by the physical instability unless appropriate precautions are taken. The most natural way to guarantee a physically meaningful, converged solution is to introduce a physically motivated dissipation length that must then be resolved on the numerical grid. When thermal conduction cannot be neglected, Field (1965) derived a stability criterion for the condensation mode of the general form $\mathrm{d}(\rho \Lambda) / \mathrm{d} T-\rho / T \mathrm{~d}(\rho \Lambda) / \mathrm{d} \rho<-k^{2} \kappa$ where $\kappa$ is the coefficient of thermal conduction in units of erg $\mathrm{cm}^{-1} \mathrm{~K}^{-1} \mathrm{~s}^{-1}$. For a power-law cooling function $\Lambda(T) \sim T^{\beta}$, this reduces to $(\beta-1) \rho^{2} \Lambda / T<-k^{2} \kappa$. The wavelength $\lambda_{\mathrm{F}}$ at which the criterion is fulfilled marginally defines the scale on which fluctuations generated by the TI are efficiently damped by thermal conduction. Koyama \& Inutsuka (2004) demonstrated that

$\lambda_{\mathrm{F}}=\frac{2 \pi}{\sqrt{1-\beta}} \sqrt{\frac{\kappa T}{\rho^{2} \Lambda}}$

must be resolved with at least three grid cells to attain a converged numerical solution. It occurs naturally to use this criterion as a condition for mesh refinement. 


\section{Numerical methods}

For our simulations, we use the NIRVANA code, which is a general-purpose MHD fluid tool designed to solve multiscale, self-gravitating hydrodynamics problems. The NIRVANA code comprises (i) a fully conservative, divergence-free Godunovtype central scheme (Ziegler 2004); (ii) a block-structured adaptive mesh refinement (AMR), and (iii) a multigrid-like adaptive mesh Poisson solver with elliptic matching (Ziegler 2005).

The central aim of adaptive mesh simulations is to resolve regions of interest at enhanced accuracy. In AMR codes such as Enzo (O'Shea et al. 2004), FLASH (Fryxell et al. 2000), NIRVANA, or RAMSES (Teyssier 2002), this can be achieved by applying a variety of different criteria: baryon or dark matter overdensity (cosmological structure formation), local Jeanslength (protostellar core collapse), or entropy gradients (accretion shocks). Iapichino et al. (2008) introduced a vorticity-based criterion to properly resolve a turbulent wake. Alternatively, and independently of the underlying physics, the amount of discrepancy between the solutions on different AMR levels can be used as an indication for refinement (Berger \& Colella 1989).

\subsection{Classical mesh refinement}

As a standard criterion for tracing developing structures, one typically monitors local gradients $\delta u$ in the fluid variables $u$. To be able to follow moving structures (and anticipate emerging structures), it is furthermore desirable to consider the temporal change in these gradients. Because of the additional overhead in storing quantities between timesteps, this is usually omitted, and one assumes that strong gradients are bordered by sections of enhanced curvature, as reflected in the second derivatives $\delta^{2} u$. Accordingly, NIRVANA's usual mesh refinement is determined by combining the criterion of normalised gradients $\|\delta u\|_{2} /|u|$ with one of the ratio of second to first derivatives $\left\|\delta^{2} u\right\|_{2} /\|\delta u\|_{2}$ of the conserved variables $u$, i.e.,

$$
\left[\alpha \frac{\|\delta u\|_{2}}{|u|}+(1-\alpha) \frac{\left\|\delta^{2} u\right\|_{2}}{\|\delta u\|_{2}+\epsilon|u|}\right]\left(\frac{\Delta x^{(l)}}{\Delta x^{(0)}}\right)^{\xi} \begin{cases}>\epsilon_{u} & \exists u \\ <0.7 \epsilon_{u} & \forall u\end{cases}
$$

where $\|\delta u\|_{2}$ and $\left\|\delta^{2} u\right\|_{2}$ are the first and second numerical derivatives in the Euclidian norm, and $\alpha \in[0,1]$ controls the mixture of the two indicators. A new block is created if the estimator exceeds a critical value $\epsilon_{u}$ in a two-cell wide buffer zone around the block to be inserted. Blocks are removed if the estimator becomes lower than a factor of 0.7 of the critical value. By tuning $\alpha$, the user can specify a bias towards the first or second type of criterion; a value of $\alpha=0$ corresponds to the standard estimator used in the FLASH code (Fryxell et al. 2000).

For our runs not based on the Field condition, we adopt refinement for the gas density $\rho$ only and use trigger values $\epsilon_{\rho}$ of 0.02 and 0.04 while we set $\alpha=0.7$. Including the ratio of grid spacings $\Delta x$ of refinement level $l$ to the base level enables a level-dependent refinement via the choice of the exponent $\xi$. Here we fix $\xi=0.6$, which makes refinements on higher levels successively easier. Finally, we set the additional filter value $\epsilon=0.01$ to prevent the refinement of small ripples.

While this general form of the refinement criterion is very flexible, the large number of free parameters renders the optimal adjustment to a particular problem a tedious task requiring a certain amount of experience. Since AMR always implies a trade-off between accuracy and efficiency, the optimal choice is also by no means clear-cut. This is illustrated in Fig. 1, where
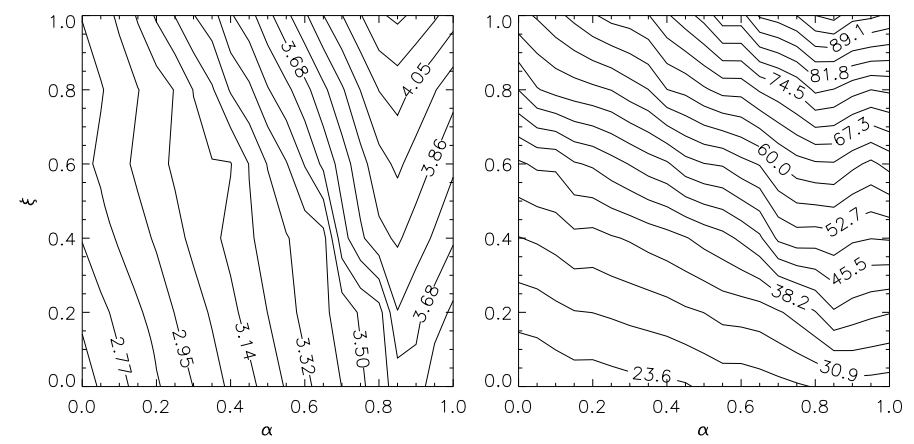

Fig. 1. Effect of the adjustable parameters $\alpha$ and $\xi$ on the accuracy/efficiency of the mesh refinement. Contour lines indicate the maximum relative error (in percent, left panel), and the corresponding AMR speed-up (right panel) for the case of $\epsilon_{\rho}=0.02$ and with respect to the fully resolved reference run.

we show the variation in the accuracy and AMR speed-up ${ }^{1}$ as a function of the two supplementary parameters $\alpha$ and $\xi$ of the classical refinement estimator (2). In principle, one can use these graphs to find the parameter set that yields the highest execution speed at a given level of fidelity. However, this procedure remains limited to simple test cases, and its universality cannot be easily proven.

\subsection{Refinement based on the Field condition}

In an alternative approach tailored to tracing condensation interfaces, we apply grid refinement wherever the local Fieldlength $\lambda_{\mathrm{F}}$ is not resolved by at least three grid cells. For fine tuning, this number can be varied as a linear function of the refinement level, although we do not use this dispensable feature here. The main advantage of the proposed new method is that it is virtually parameter free. This implies that the intricate determination of suitable values for $\alpha$ and $\xi$ (as described above) can be avoided.

The evaluation of $\lambda_{\mathrm{F}}$, given by Eq. (1), is straightforward since the values of $\kappa$ and $T$ have already been computed for the heat flux term. To minimise overheads, they are stored in auxiliary arrays. It is evident that the definition of the Field-length requires the inclusion of a heat conduction term in the energy equation. In contrast to this, the FLASH code supports refinement based on the local cooling time $\tau=\varepsilon / \rho^{2} \Lambda$, which is independent of $\kappa$. In our case, if $\kappa$ is scaled by $\rho$ (see below), the two methods should perform similarly.

\section{Results}

\subsection{Gaussian density perturbation}

As a test problem, we revisit the 1D case of a density perturbation as considered in the model DEN3 from Sánchez-Salcedo et al. (2002, hereafter SSVSG). In this setup, a gas of density $n=1 \mathrm{~cm}^{-3}$ initially rests at its equilibrium pressure of $p / k_{\mathrm{B}}=2500 \mathrm{~K} \mathrm{~cm}^{-3}$ and is perturbed by a Gaussian overdensity of amplitude $0.2, F W H M$ of 3 pc, and centred within a domain of 16 pc. Unlike in our simulations, SSVSG do not apply thermal conduction. This means that we must apply a high enough resolution so that conduction (which becomes important mainly on the grid scale) does not affect significantly the features seen in their solution. As a reference, we perform a uni-grid run at a

\footnotetext{
${ }^{1}$ For a description of the test case, see Sect. 3.1 below.
} 


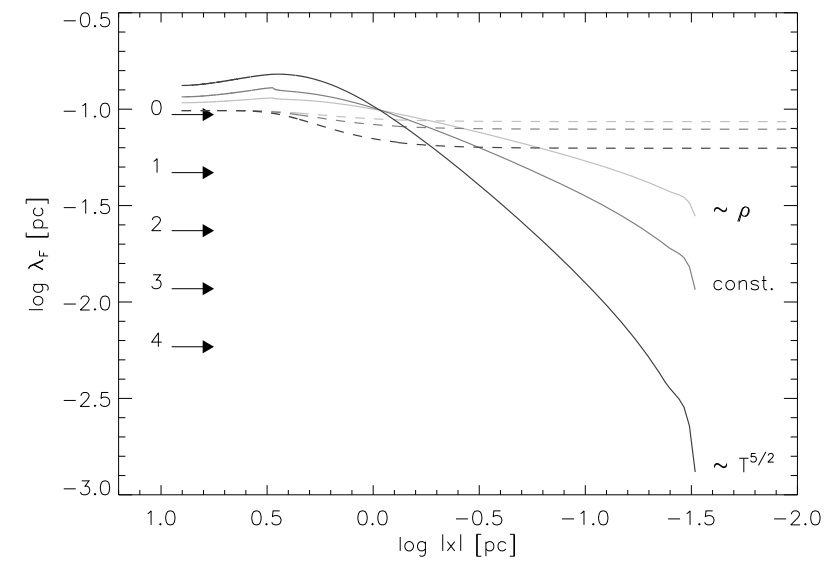

Fig. 2. Field-length as a function of radius for the problem DEN3 described in Sánchez-Salcedo et al. (2002) for the initial profile (dashed lines), and the final profile at $t=4.2 \mathrm{Myr}$ (solid lines). The diffusion is set at a level of $1.02 \times 10^{4} \mathrm{erg} \mathrm{cm}^{-1} \mathrm{~K}^{-1} \mathrm{~s}^{-1}$ and scaled as indicated by the labels. Arrows mark a spacing of $3 \Delta x$ at different refinement levels.

linear resolution of 8192 cells, corresponding to a grid spacing of $\simeq 2 \times 10^{-3} \mathrm{pc}$. The level of conduction is then chosen in such a way that $\lambda_{\mathrm{F}}$ is resolved properly in the reference run. This yields a value for $\kappa$ which is about a factor of 500 higher than the physical Spitzer value in the limit of vanishing electron density. Thus, the even smaller structures expected in a physically meaningful scenario illustrate the need for applying multiscale techniques.

Figure 2 shows a plot of the Field-length for the initial and final density and temperature profiles, where we use three different prescriptions for the conduction. Scaling $\kappa$ by $\rho$ is typically used in combination with a constant kinematic viscosity yielding a constant value for the Prandtl number and a constant numerical time step, which is computationally favourable. This is also reflected in the Field-length varying only weakly with density. For the case of constant $\kappa$, this variation is stronger and accounts for about one order of magnitude in the problem under consideration. Finally, for a Spitzer-type conductivity of $\kappa \sim T^{5 / 2}$, we obtain a variation of over two orders of magnitude in $\lambda_{F}$ rendering this case particularly interesting for AMR. In the following, we restrict ourselves to the case of a constant coefficient $\kappa=1.02 \times 10^{4} \mathrm{erg} \mathrm{cm}^{-1} \mathrm{~K}^{-1} \mathrm{~s}^{-1}$.

In general, the results are only marginally affected when conduction is included and agree well with the solution of SSVSG. In Fig. 3, the resulting profiles for the run with a 512 cell basegrid plus 4 levels of refinement (based on the Field condition) are plotted. Before we proceed with an analysis of the AMR performance, we want to compare our reference run $8192+0$ (which is barely distinguishable from the one shown in Fig. 3) with the profiles from SSVSG: the most noticeable discrepancy is in the overall level of the thermal pressure. Due to the assumed periodicity at the domain boundaries, the mean pressure becomes a highly fluctuating quantity (cf. central panel of Fig. 3). Apart from the different offsets, the pressure profiles match reasonably well. We note that there is a small pressure dip at the interface of the condensed structure in our results that is not seen in SSVSG. A similar feature is observed in panel (d) of their Fig. 7 (model DEN75), although much spikier. This leaves the possibility that the feature is not resolved by the plot for model DEN3 in Fig. 2 of SSVSG. A comparison run with $\kappa=0$ in fact shows that, in our setup, the dip is broadened by the thermal conduction and otherwise remains much narrower. Later investigations of the topic consistently reveal a similar region of lower pressure (see e.g., Vázquez-Semadeni et al. 2003), which can be attributed to the pressure decreasing with density in the unstable regime. In this respect, the pressure dip reflects the S-shape of the equilibrium cooling curve.

In Fig. 4, we plot the relative errors of three AMR runs with respect to the fully resolved solution. The two panels correspond to the two intervals with the strongest deviations, i.e., an outgoing wave (upper panel) and the condensation interface of the cloud (lower panel). We compare the two runs based on the density gradient criterion (with $\epsilon_{\rho}=0.02$ and 0.04 ) with the run based on the Field condition. At the time $t=4.2 \mathrm{Myr}$, the number of AMR blocks for these runs are 820, 476, and 504, respectively. Although the number of refined cells is comparable, the Field condition yields a relative error that is lower by a factor of two at the condensation front. For $\epsilon_{\rho}=0.04$ (0.02), the outgoing wave is resolved at $l=1$ (3), while the Field condition, as expected, is insensitive to this feature and does not trigger refinement at all. Yet the relative error is the lowest in this case, indicating that the wave might be overly steepened by the refinement based on the density gradients. This finding is rather surprising and requires further investigation.

\section{Discussion}

As illustrated in Fig. 5, thermal fragmentation produces turbulent and extremely filamentary structures. Since turbulence can be modelled properly only in three dimensions, adaptive mesh refinement becomes highly beneficial, if not mandatory. Because classical refinement strategies reach their limits if turbulence is involved (see e.g., Iapichino et al. 2008, for a vorticity-based refinement criterion), one must seek new tracers of structural properties. This is particularly true in the case of a thermally unstable medium.

Until now, the criterion introduced by Koyama \& Inutsuka (2004) has been widely disregarded by many members of the numerical astrophysics community. Notable exceptions are a TI study by Brandenburg et al. (2007) and the MRI simulations of Piontek \& Ostriker (2004). The opposite standpoint, represented by a number of authors (see e.g., Gazol et al. 2005; Joung \& Mac Low 2006), is to neglect thermal conduction altogether. The standard argument is that numerical diffusion defines a "numerical Field-length" that is believed to damp down smallscale modes of the instability sufficiently. On the other hand, de Avillez \& Breitschwerdt (2004) argued that microscopic heat conduction is suppressed perpendicular to the magnetic field lines (and thus also isotropically for sufficiently tangled fields) and that turbulent transport plays an important role. Although this is certainly true, it does not aid the discussion of whether explicit conduction should be included.

In our own simulations, we observe artificial growth of modes close to the resolution limit only in situations where the Courant number is chosen to be inappropriately high or the gradient-based refinement criterion is adjusted improperly. The absence of unphysical growth at high wavenumbers might thus, in fact, be attributed to the finite level of numerical dissipation, which is never really negligible in $3 \mathrm{D}$ simulations.

In this respect, the Field criterion of Koyama \& Inutsuka appears to be of academic interest only. Apart from its true necessity, we have however demonstrated that the condition can be used successfully as a refinement criterion for adaptive mesh simulations of the interstellar medium, an approach that is similar to the refinement procedure based on the Jeans-length introduced by Truelove et al. (1997) for self-gravitating clouds. In a simple $1 \mathrm{D}$ test case, the new refinement estimator is found to 

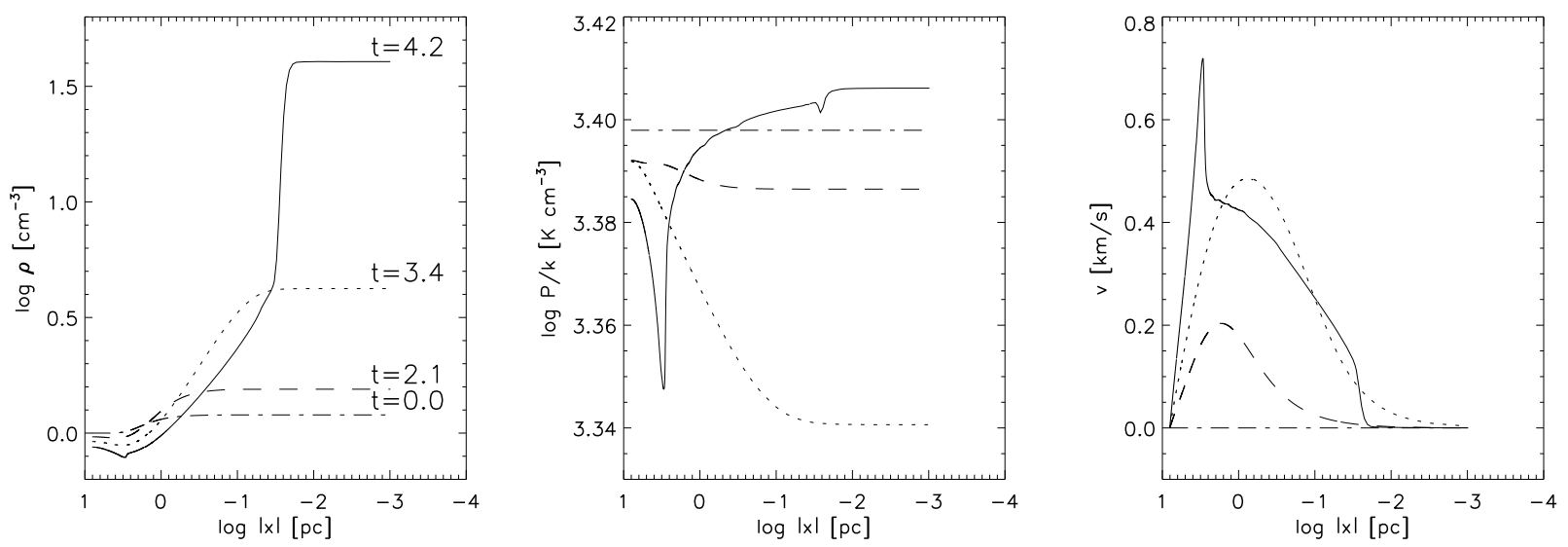

Fig. 3. Temporal evolution of density (left), thermal pressure (centre), and velocity (right), for our $512+4$ run with constant $\kappa$ and refinement based on the Field condition (same as Fig. 2 of Sánchez-Salcedo et al. 2002).
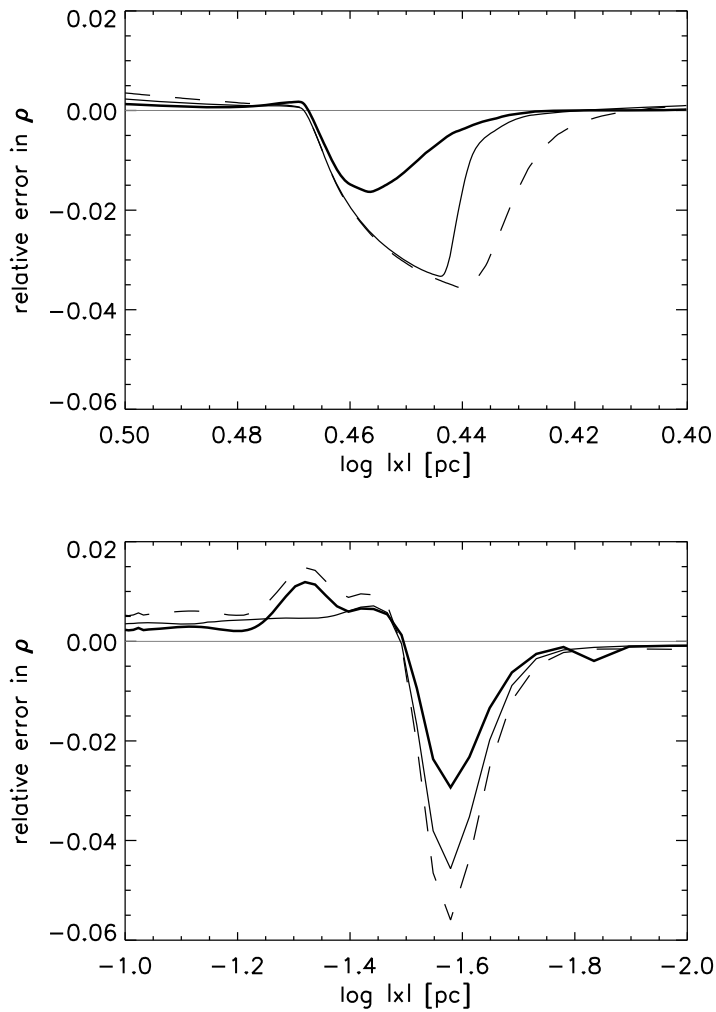

Fig. 4. Relative errors of the density profiles at time $t=4.2 \mathrm{Myr}$ with respect to the fully resolved reference solution. The two intervals with the highest deviations are shown for refinement based on $\lambda_{\mathrm{F}}$ (thick line), $\varepsilon_{\rho}=0.02$ (solid line), and $\varepsilon_{\rho}=0.04$ (dashed line).

produce more accurate results at comparable numerical cost than conventional criteria.

The overhead of evaluating $\lambda_{\mathrm{F}}$ is low compared to the computation of the numerical fluxes. Within NIRVANA, the determination of the heat flux already requires the evaluation of the gas temperature and the (non-uniform) conductivity coefficient. These fields can therefore be stored temporarily such that the only additional expense comes from evaluating the cooling function.

The definition of $\lambda_{F}$ nevertheless implies the inclusion of thermal conduction, which is not a standard feature in many available codes. An alternative approach (implemented in the FLASH code) is instead to use the cooling time, which is

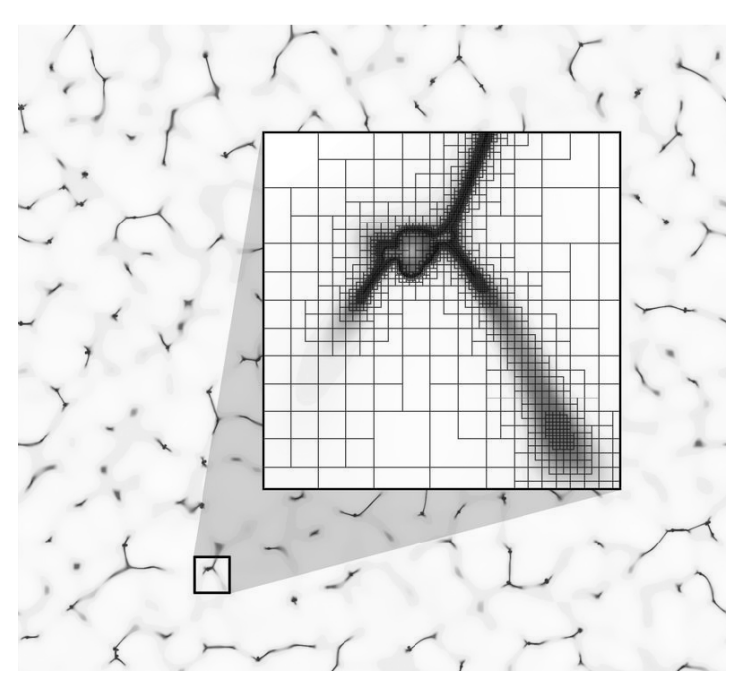

Fig. 5. The Field-length criterion in action: development of the thermal instability from random fluctuations with Spitzer-type conductivity. The 2D simulation has a base grid of $256^{2}$ with refinement of up to 8 levels, covering scales from $0.003-200 \mathrm{pc}$.

independent of $\kappa$. On the other hand, there is evidence (Piontek, in prep.) that heat conduction is in fact physically relevant to the formation of molecular clouds, providing further motivation for the use of the proposed scheme.

Compared to classical mesh refinement based on gradients, which has to be fine-tuned by multiple parameters according to different situations, the Field criterion is virtually parameter-free and provides reliable results irrespective of the particular setup. If it becomes mandatory to adequately resolve features such as shock fronts, one must, of course, still rely on a combination with conventional refinement criteria. This does, however, not impose any difficulties.

Acknowledgements. This work made use of the NIRVANA code developed by Udo Ziegler at the Astrophysical Institute Potsdam. I thank Enrique VázquezSemadeni and Simon Glover for discussions related to this work and acknowledge the helpful comments by the anonymous referee.

\section{References}

Audit, E., \& Hennebelle, P. 2005, A\&A, 433, 1

Berger, M. J., \& Colella, P. 1989, JCoPh, 82, 64

Brandenburg, A., Korpi, M. J., \& Mee, A. J. 2007, ApJ, 654, 945

de Avillez, M. A., \& Breitschwerdt, D. 2004, A\&A, 425, 899 
de Avillez, M. A. \& Breitschwerdt, D. 2005, A\&A, 436, 585

Field, G. B. 1965, ApJ, 142, 531

Fryxell, B., Olson, K., Ricker, P., et al. 2000, ApJS, 131, 273

Gazol, A., Vázquez-Semadeni, E., \& Kim, J. 2005, ApJ, 630, 911

Gressel, O., Ziegler, U., Elstner, D., \& Rüdiger, G. 2008a, AN, 329, 619

Gressel, O., Elstner, D., Ziegler, U., \& Rüdiger, G. 2008b, A\&A, 486, L35

Heitsch, F., Hartmann, L. W., \& Burkert, A. 2008, ApJ, 683, 786

Hennebelle, P., Banerjee, R., Vázquez-Semadeni, E., Klessen, R. S., \& Audit, E. 2008, A\&A, 486, L43

Iapichino, L., Adamek, J., Schmidt, W., \& Niemeyer, J. C. 2008, MNRAS, 388, 1079

Joung, M. K. R., \& Mac Low, M.-M. 2006, ApJ, 653, 1266

Korpi, M. J., Brandenburg, A., Shukurov, A., Tuominen, I., \& Nordlund, Å 1999, ApJ, 514, L99

Koyama, H., \& Inutsuka, S.-i. 2004, ApJ, 602, L25

Koyama, H., \& Inutsuka, S.-i. 2006 [arXiv: astro-ph/0605528]
O'Shea, B. W., Bryan, G., Bordner, J., et al. 2004 [arXiv: astro-ph/0403044] Piontek, R. A., \& Ostriker, E. C. 2004, ApJ, 601, 905

Piontek, R. A., \& Ostriker, E. C. 2005, ApJ, 629, 849

Piontek, R. A., \& Ostriker, E. C. 2007, ApJ, 663, 183

Sánchez-Salcedo, F. J., Vázquez-Semadeni, E., \& Gazol, A. 2002, ApJ, 577, 768

Teyssier, R. 2002, A\&A, 385, 337

Truelove, J. K., Klein, R. I., McKee, C. F., et al. 1997, ApJ, 489, 179

Vázquez-Semadeni, E., Gazol, A., \& Passot, T. 2003, in Turbulence and Magnetic Fields in Astrophysics, ed. E. Falgarone, \& T. Passot, Lect. Notes in Phys. (Berlin: Springer Verlag), 614, 213

Vázquez-Semadeni, E., Ryu, D., Passot, T., González, R. F., \& Gazol, A. 2006, ApJ, 643, 245

Wolfire, M. G., Hollenbach, D., McKee, C. F., Tielens, A. G. G. M., \& Bakes, E. L. O. $1995, \mathrm{ApJ}, 443,152$

Ziegler, U. 2004, JCoPh, 196, 393

Ziegler, U. 2005, CoPhC, 170, 153 